\title{
Type 2 diabetes and risk of non-embolic ischaemic stroke in Japanese men and women
}

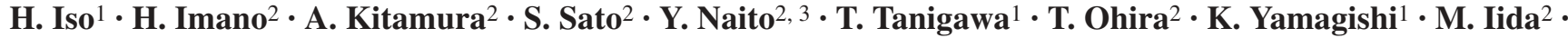 \\ T. Shimamoto ${ }^{2}$ \\ ${ }^{1}$ Department of Public Health Medicine, Social and Environmental Medicine Doctoral Program, Graduate School \\ of Comprehensive Human Sciences, University of Tsukuba, Ibaraki-ken, Japan \\ 2 The Osaka Medical Center for Health Science and Promotion, Osaka, Japan \\ ${ }^{3}$ Department of Nutrition and Food Sciences, Mukogawa Women's University, Nishinomiya, Japan
}

\section{Abstract}

Aims/hypothesis. The aim of this study was to examine the relationship between type 2 diabetes and risk of ischaemic stroke in Asian populations.

Methods. We conducted a 17-year prospective cohort study in 10,582 Japanese individuals (4287 men and 6295 women) aged 40-69 years living in five communities in Japan. All subjects were free of stroke and CHD at baseline. Diabetes was defined as a fasting glucose level of $\geq 7.0 \mathrm{mmol} / \mathrm{l}$, a non-fasting glucose of $\geq 11.1 \mathrm{mmol} / 1$, or receiving medication for diabetes.

Results. The risk of non-embolic ischaemic stroke was approximately two-fold higher in diabetic subjects than in subjects with normal glucose levels. The multivariate relative risk after adjustment for age, community, hypertensive status, BMI, triceps and subscapular skinfold thickness (TSF and SSF), and other known cardiovascular risk factors was 1.8 (95\% CI 1.0-3.2) for men and $2.2(1.2-4.0)$ for women. This excess risk was primarily observed among non-hypertensive subjects and individuals with higher values for measures of adiposity (BMI, TSF and SSF values above the median), particularly those with higher values for SSF. The association between non-embolic ischaemic stroke and glucose abnormality was particularly strong among nonhypertensive subjects with higher SSF values: the multivariate relative risk was $1.9(1.0-3.7)$ for borderline diabetes and 4.9 (2.5-9.5) for diabetes.

Conclusions/interpretation. In this cohort, type 2 diabetes was a significant risk factor for non-embolic ischaemic stroke, particularly in non-hypertensive and non-lean individuals. Due to the nationwide decrease in blood pressure and increase in mean BMI among the Japanese population, with current levels approaching those observed in Western countries, the impact of glucose abnormalities on risk of ischaemic stroke represents a forthcoming public health issue in Japan.

Keywords Adiposity · BMI · Follow-up studies · Ischaemic stroke - Japanese - Type 2 diabetes . Skinfold thickness.
Received: 10 May 2004 / Accepted: 19 July 2004

Published online: 15 December 2004

C) Springer-Verlag 2004

H. Iso ( $)$

Department of Public Health Medicine,

Social and Environmental Medicine Doctoral Program,

Graduate School of Comprehensive Human Sciences,

University of Tsukuba, 1-1-1, Tsukuba-shi,

Ibaraki-ken 305-8575, Japan

E-mail: h-iso@md.tsukuba.ac.jp

Tel.: +81-298-532695, Fax: +81-298-532695

Abbreviations: CT, computerised tomography $\cdot \mathrm{MRI}$, magnetic resonance imaging $\cdot \mathrm{RR}$, relative risk $\cdot \mathrm{SSF}$, subscapular skinfold thickness $\cdot$ TSF, triceps skinfold thickness

\section{Introduction}

Previous studies in Western countries and Japanese Americans have shown that diabetes, IFG and IGT are significant risk factors for ischaemic stroke [1, 2, 3]. However, data on Japanese people living in Japan and on other Asian populations are extremely limited [4]. Due to the lower prevalence of diabetes in Asia compared with that in Western countries [5], the majority of existing cohort studies in Asia may have a low statistical power for detecting an association between diabetes and risk of ischaemic stroke.

Since the 1960s, the prevalence of diabetes [5] and the mean BMI had increased in Japan, while blood 
pressure levels have decreased [6]. Because hypertension is a primary risk factor for ischaemic stroke $[1$, 4], we postulated that the increased prevalence of diabetes and obesity and the decreased blood pressure levels may increase the relative contribution of diabetes to the development of ischaemic stroke. To investigate whether diabetes is a significant predictor of ischaemic stroke in Japanese men and women, particularly in non-hypertensive and non-lean individuals, we analysed data from a large prospective study of 10,000 middle-aged men and women in five Japanese communities.

\section{Subjects and methods}

Study population. The study population included residents of five communities who participated in one of three cardiovascular risk surveys. The first was performed between 1975 and 1980 in two northeast rural communities (the towns of Ikawa and Ishizawa, Akita Prefecture) and in a southwest rural community (the town of Noichi, Kochi Prefecture). The second was carried out between 1981 and 1986 in a central rural community (the town of Kyowa, Ibaraki Prefecture). The third was conducted between 1975 and 1984 in a southwest urban suburb (the city of Yao, Osaka Prefecture). Subjects were aged 40-69 years, and the overall participation rate at baseline was $67 \%$. After excluding individuals with a history of CHD $(n=66)$ and stroke $(n=135)$ were excluded, 10,582 individuals (4287 men and 6295 women) remained in the analyses. Subjects were followed-up through 2000 to determine the incidence of stroke endpoints. A total of $632(6 \%)$ subjects moved out of the communities during the follow-up period, as determined by the municipal office records on emigration, and 1750 (17\%) died. For these subjects, follow-up was censored at the date of moving out or the date of death. The average follow-up period was 16.9 years. Informed consent was obtained from the participants and the study was approved by the Ethics Committee of the University of Tsukuba.

Endpoint determination. The participants were followed-up to identify the number of incident strokes that occurred up to the end of 2000. The details of endpoint determination have been previously described elsewhere [7]. Briefly, to obtain histories of incident strokes, participants completed annual cardiovascular risk surveys. For subjects who had moved from the community or died, the incidence of stroke was determined by mailed questionnaire and by the use of death certificates respectively. Cases with stroke as an underlying cause of death (International Classification of Diseases, 9th Revision) were selected from the death certificates. We also used national insurance claims, ambulance records, reports by local physicians, and reports by public health nurses and health volunteers. To confirm the diagnosis, all living patients were either telephoned or visited to obtain their medical history, and their medical records were reviewed. For deaths, we obtained histories from families and reviewed medical records.

Stroke was defined as a focal neurological disorder with rapid onset, persisting for at least $24 \mathrm{~h}$ or until death. Using the clinical criteria, incident strokes were identified by a panel of three to four study physicians who were blinded to the data from the risk factor survey. Stroke events were classified as intraparenchymal haemorrhages, subarachnoid haemorrhages, ischaemic strokes (thrombotic or embolic), or strokes of undetermined type, primarily based on computerised tomography
(CT) and/or magnetic resonance imaging (MRI) [8]. Nonembolic ischaemic strokes were subclassified as lacunar infarctions or large-artery occlusive infarctions [8].

In the present study, the endpoints of interest were non-embolic ischaemic stroke and its subtypes. Embolic infarction was not included because atrial fibrillation, which is relevant to chronic hypertension but not glucose abnormality, was the embolic source in the majority of cases [9].

Baseline examination. At baseline (study entry), blood was drawn from seated participants into a plain, siliconised glass tube and the serum was separated. Fasting was not required prior to blood draw. The distribution of time since the last meal was: $<2 \mathrm{~h}, 39 \%$; $2 \mathrm{~h}, 43 \% ; 3-7 \mathrm{~h}, 9 \%$; and $\geq 8$ h, 9\%. Serum glucose was measured by the cupric-neocuproine method between 1975 and September 1986 and by the hexokinase method thereafter. Glucose values obtained using the first method were adjusted using the formula of the regression line estimated from 60 random samples of blood: $0.047 \times$ (glucose concentration in $\mathrm{mmol} / \mathrm{l})-0.541, r^{2}=0.93$. Glucose values were subsequently divided into three categories (normal, borderline diabetes and diabetes) according to modified criteria of the Japan Diabetes Society [10]. Borderline diabetes was defined as a fasting glucose level of $6.1-6.9 \mathrm{mmol} / \mathrm{l}$ or a non-fasting glucose level of 7.8-11.0 mmol/l, without the use of medication for diabetes. Diabetes was defined as a fasting glucose level of $\geq 7.0 \mathrm{mmol} / \mathrm{l}$, a non-fasting glucose level of $\geq 11.1 \mathrm{mmol} / \mathrm{l}$, or the use of medication for diabetes. Unlike the original criteria [10] and those of American Diabetic Association [11], we relied on a single measurement and did not require the presence of symptoms (polyuria, polydipsia and unexplained weight loss) for the diagnosis of individuals with a non-fasting glucose level of $\geq 11.1 \mathrm{mmol} / \mathrm{l}$, due to the fact that the symptoms could not be reliably assessed in the survey situation.

Serum total cholesterol was measured by the Liebermann-Burchard direct method using the Autoanalyzer II (Technicon, Tarrytown, N.Y., USA) at the Osaka Medical Center for Cancer and Cardiovascular Diseases (presently the Osaka Medical Center for Health Science and Promotion). The Osaka laboratory has been standardised by the Centers for Disease Control / National Heart, Lung, and Blood Institute (CDC-NHLBI) Lipid Standardization Program, and successfully met the criteria for precision and accuracy of triglyceride and cholesterol measurements [12].

Blood pressures were measured by trained observers using standard mercury sphygmomanometers on the right arm of seated participants after a $5 \mathrm{~min}$ rest [13]. Hypertension was defined as a systolic blood pressure $\geq 160 \mathrm{~mm} \mathrm{Hg}$, a diastolic blood pressure $\geq 95 \mathrm{~mm} \mathrm{Hg}$, or use of antihypertensive medication, while normotension was defined as a systolic blood pressure $<140 \mathrm{~mm} \mathrm{Hg}$ and a diastolic blood pressure $<90 \mathrm{~mm} \mathrm{Hg}$ without the use of antihypertensive medication. Subjects who did not meet either criterion were classified as having borderline hypertension. Height in stockinged feet and weight in light clothing were measured. BMI was calculated as weight $(\mathrm{kg})$ / [height $(\mathrm{m})]^{2}$. Triceps skinfold thickness (TSF) and subcapular skinfold thickness (SSF) were measured to the nearest mm using Keys calipers. An interview was conducted to determine the number of cigarettes smoked per day, usual weekly intake of alcohol in go (a Japanese traditional unit of volume corresponding to $23 \mathrm{~g}$ of ethanol), and the menopausal status of the female subjects.

Statistical analysis. Due to the fact that a previous Japanese study [4] reported that the relationship between diabetes and risk of ischaemic stroke varied according to sex, we conducted sex-specific analyses in addition to sex-adjusted analyses. 
Table 1. Age- and community-adjusted mean values or prevalences of risk factors at baseline according to serum glucose category

\begin{tabular}{|c|c|c|c|c|c|c|c|c|}
\hline & \multicolumn{4}{|l|}{ Men } & \multicolumn{4}{|l|}{ Women } \\
\hline & $\begin{array}{l}\text { Normal } \\
(n=3737)\end{array}$ & $\begin{array}{l}\text { Borderline } \\
(n=411)\end{array}$ & $\begin{array}{l}\text { Diabetes } \\
(n=139)\end{array}$ & $\begin{array}{l}p \text { value for } \\
\text { difference }\end{array}$ & $\begin{array}{l}\text { Normal } \\
(n=5882)\end{array}$ & $\begin{array}{l}\text { Borderline } \\
(n=285)\end{array}$ & $\begin{array}{l}\text { Diabetes } \\
(n=128)\end{array}$ & $\begin{array}{l}p \text { value for } \\
\text { difference }\end{array}$ \\
\hline Age (years) & 53 & 53 & 55 & 0.04 & 53 & 56 & 58 & $<0.001$ \\
\hline Serum glucose $(\mathrm{mmol} / \mathrm{l})^{\mathrm{a}}$ & 5.42 & 8.54 & 12.1 & $<0.001$ & 5.25 & 8.52 & 13.0 & $<0.001$ \\
\hline Systolic blood pressure (mm Hg) & 137 & 141 & 141 & $<0.001$ & 134 & 139 & 140 & $<0.001$ \\
\hline Hypertensive (\%) & 28 & 30 & 36 & 0.07 & 23 & 29 & 31 & 0.004 \\
\hline $\mathrm{BMI}\left(\mathrm{kg} / \mathrm{m}^{2}\right)$ & 22.8 & 23.1 & 23.3 & 0.02 & 23.4 & 23.9 & 25.0 & $<0.001$ \\
\hline $\mathrm{TSF}(\mathrm{mm})$ & 7.3 & 8.3 & 7.8 & $<0.001$ & 15.6 & 16.5 & 16.4 & 0.02 \\
\hline $\mathrm{SSF}(\mathrm{mm})$ & 12.1 & 13.1 & 12.9 & 0.001 & 18.5 & 20.1 & 21.5 & $<0.001$ \\
\hline Serum total cholesterol (mmol/l) & 4.68 & 4.79 & 4.78 & 0.03 & 5.00 & 5.23 & 5.20 & $<0.001$ \\
\hline HDL cholesterol (mmol/l) & 1.45 & 1.44 & 1.45 & 0.86 & 1.48 & 1.48 & 1.39 & 0.08 \\
\hline
\end{tabular}

a Also adjusted for time since last meal

Age-adjusted mean values and the prevalence rates of covariates were calculated according to serum glucose category, and differences between categories were tested by analysis of covariance or the chi square test.

Person-years were calculated as the sum of individual follow-up time until the occurrence of incident stroke, death, emigration or the end of the year 2000. The Cox proportional hazards model was used to calculate the relative risks of ischaemic stroke and their respective $95 \%$ confidence intervals, using the risk of individuals in the normal glucose category as a reference value. A test for trend between the serum glucose category and risk of ischaemic stroke was also conducted by applying sex-specific median glucose values, adjusted for age, sex, time since last meal and community, to each glucose category. We adjusted for age and community, and for other potential confounding variables including hypertension status, sexspecific quartiles of BMI, triceps skinfold thickness (TSF), subscapular skinfold thickness (SSF), serum total and HDL cholesterol, smoking status, alcohol intake category, and for the menopausal status of women. We conducted stratified analyses by hypertension status, BMI, TSF and SSF to assess potential effect modification. The significance of the interaction between serum glucose category and hypertension status and adiposity was tested using cross-product terms of these variables in multivariate models. All analyses were conducted using the SAS statistical package, Version 8 (SAS Institute, Cary, N.C., USA). All $p$ values for statistical tests were two-tailed, and a $p$ value less than 0.05 was regarded as statistically significant.

\section{Results}

By the end of 2000 , representing 178,828 personyears of follow-up, we had documented 400 incident non-embolic ischaemic strokes (219 in men, 181 in women; $80 \%$ confirmed by CT or MRI), 144 intraparenchymal haemorrhages (69 in men, 75 in women), 89 subarachnoid haemorrhages (30 in men, 59 in women), 39 embolic strokes (20 in men, 19 in women), and 36 unclassified strokes (17 in men, 19 in women). Among the non-embolic ischaemic strokes we identified 254 lacunar strokes (131 in men, 123 in women) and 60 large-artery occlusive infarctions (33 in men, 27 in women); the remaining 86 cases (55 in men, 31 in women) were not further classified due to a lack of imaging studies.

Table 1 shows the age-adjusted means and percentages of selected cardiovascular risk factors at baseline according to serum glucose category. The prevalence of borderline diabetes was $9.6 \%$ among the men and $4.5 \%$ among the women, and the corresponding values for diabetes were $3.2 \%$ and $2.0 \%$ respectively. Abnormal glucose levels were positively associated with age, systolic and diastolic blood pressure, hypertension, BMI, and serum total cholesterol levels in both sexes. Glucose abnormality was positively associated with ethanol intake in men alone and was inversely associated with HDL cholesterol levels in women alone.

Compared with those in the normal glucose category, the age- and community-adjusted risk of non-embolic ischaemic stroke was two-fold higher in men in the diabetic category and two-and-half-fold higher in women in the diabetic category (Table 2). We observed a dose-response relationship between serum glucose category and the risk of ischaemic stroke among the women. Further adjustment for known cardiovascular risk factors reduced the excess risk of ischaemic stroke with diabetes; however, the association remained statistically significant for both sexes. Lacunar infarction comprised $64 \%$ of non-embolic ischaemic strokes and was similarly associated with glucose abnormality in terms of risk. Analysis of the combined data for men and women revealed that serum glucose category was not significantly associated with the risk of intraparenchymal haemorrhage (multivariate relative risk $[R R]=0.90,95 \%$ CI $0.33-2.46$, 
Table 2. Serum glucose category and sex-specific risk of non-embolic ischaemic stroke

\begin{tabular}{|c|c|c|c|c|c|c|c|c|}
\hline & \multicolumn{4}{|l|}{ Men } & \multicolumn{4}{|l|}{ Women } \\
\hline & Normal & Borderline & Diabetes & $\begin{array}{l}p \text { value } \\
\text { for trend }\end{array}$ & Normal & Borderline & Diabetes & $\begin{array}{l}p \text { value } \\
\text { for trend }\end{array}$ \\
\hline Person-years & 60,800 & 6730 & 1947 & & 102,476 & 4860 & 2015 & \\
\hline \multicolumn{9}{|l|}{ Ischaemic stroke } \\
\hline Multivariate RR $(95 \% \mathrm{CI})^{\mathrm{a}}$ & 1.0 & $0.9(0.6-1.5)$ & $1.8(1.0-3.2)$ & 0.14 & 1.0 & $1.3(0.8-2.3)$ & $2.2(1.2-4.0)$ & 0.008 \\
\hline \multicolumn{9}{|l|}{ Lacunar infarction } \\
\hline No. of cases & 109 & 14 & 8 & & 104 & 11 & 8 & \\
\hline $\begin{array}{l}\text { Age- and community- } \\
\text { adjusted RR (95\% CI) }\end{array}$ & 1.0 & $1.2(0.7-2.0)$ & $2.1(1.0-4.2)$ & 0.07 & 1.0 & $1.6(0.9-3.0)$ & $2.8(1.4-5.8)$ & 0.002 \\
\hline
\end{tabular}

a Adjusted for age (5-year categories), community, hypertension status (normotension, borderline hypertension and hypertension), sex-specific quartiles of BMI $\left(\mathrm{kg} / \mathrm{m}^{2}\right)$, TSF $(\mathrm{mm})$, SSF $(\mathrm{mm})$, serum total cholesterol $(\mathrm{mmol} / \mathrm{l})$ and HDL choles-

$p=0.39$ for trend) or subarachnoid haemorrhage ( $\mathrm{RR}=0.76,95 \%$ CI $0.19-3.12, p=0.89$ for trend).

The relationship between blood glucose category stratified according to hypertension status or BMI and risk of non-embolic ischaemic stroke is shown in Table 3. The association between the risk of ischaemic stroke and diabetes was primarily observed in non-hypertensive subjects. The excess risk of ischaemic stroke was greater in individuals with higher adiposity (BMI, TSF and SSF values above the median), particularly those with higher SSF values. The interaction with glucose category was statistically significant for hypertension status $(p=0.006)$ and for $\operatorname{SSF}(p=0.02)$, of borderline significance for TSF $(p=0.07)$, and was not significant for BMI $(p=0.97)$. Similar associations were found between glucose category stratified by hypertension status, BMI, TSF or SSF and risk of lacunar infarction (data not shown).

The multivariate relative risks of non-embolic ischaemic stroke associated with glucose category stratified by hypertension status and adiposity combined are shown in Table 3. To analyse the combined effects of glucose abnormality, hypertension and adiposity on the risk, we also determined the age- and sex-adjusted incidence (per 1000 person-years) of non-embolic ischaemic stroke in each category (Fig. 1). There was a strong association between glucose abnormality and risk of non-embolic ischaemic stroke among non-hypertensive subjects with higher values for measures of adiposity. The calculated relative risks were 3.6 (95\% CI 1.8-7.4), 3.6 (95\% CI 1.7-7.4) and 4.9 (95\% CI 2.5-9.5) for non-hypertensive diabetic subjects with higher BMI, higher TSF and higher SSF values respectively.

The varied association between the glucose category subdivided according to hypertension/adiposity and terol (mmol/l), smoking status (never, former and current: $1-19$ and $\geq 20$ cigarettes per day), alcohol intake (never, former, and current: $<46,46-68$ and $\geq 69 \mathrm{~g}$ /day ethanol) and, for women, menopausal status (pre- and post-menopause)

the risk of non-embolic ischaemic stroke was paralleled by the mean glucose levels in three glucose categories. In diabetic subjects, the mean glucose value $( \pm$ SEM) adjusted for sex, age, time since last meal and community was $12.8 \pm 0.1 \mathrm{mmol} / \mathrm{l}$ in non-hypertensive subjects with higher BMI values, $12.2 \pm 0.1 \mathrm{mmol} / 1$ in non-hypertensive subjects with lower BMI values, $12.5 \pm 0.1 \mathrm{mmol} / \mathrm{l}$ in hypertensive subjects with higher BMI values, and $12.4 \pm 0.2 \mathrm{mmol} / \mathrm{l}$ for hypertensive subjects with lower BMI values $(p<0.001$ for difference between groups). The respective glucose values for hypertension and TSF categories were $12.8 \pm 0.1 \mathrm{mmol} / \mathrm{l}, 12.2 \pm 0.1 \mathrm{mmol} / \mathrm{l}, 12.4 \pm 0.1 \mathrm{mmol} / 1$ and $12.5 \pm 0.2 \mathrm{mmol} / \mathrm{l}(p<0.001$ for difference between groups), and those for hypertension and SSF categories were $12.5 \pm 0.1 \mathrm{mmol} / 1, \quad 12.6 \pm 0.1 \mathrm{mmol} / \mathrm{l}$, $12.6 \pm 0.1 \mathrm{mmol} / \mathrm{l}$ and $12.0 \pm 0.2 \mathrm{mmol} / \mathrm{l}(p=0.01$ for difference between groups).

\section{Discussion}

In the present study, the age-adjusted risk of nonembolic ischaemic stroke, more specifically lacunar stroke, was two-fold higher in Japanese diabetic patients (both men and women) than in individuals with normal glucose levels. This excess risk remained statistically significant for both sexes after further adjustment for established cardiovascular risk factors. The magnitude of the excess risk of ischaemic stroke associated with diabetes was similar to that reported in previous studies in Western countries ( $R R=1.5-3.6)[1,2,3]$.

The excess risk of ischaemic stroke associated with diabetes was primarily observed in non-hypertensive subjects and those with higher adiposity, particularly 
Table 3. Serum glucose category stratified by hypertension status and adiposity, separately and combined, and risk of non-embolic ischaemic stroke for the study population

\begin{tabular}{|c|c|c|c|c|c|c|c|c|}
\hline & Normal & Borderline & Diabetes & $\begin{array}{l}p \text { for } \\
\text { trend }\end{array}$ & Normal & Borderline & Diabetes & $\begin{array}{l}p \text { for } \\
\text { trend }\end{array}$ \\
\hline & \multicolumn{4}{|c|}{ Non-hypertensive } & \multicolumn{4}{|c|}{ Hypertensive } \\
\hline Person-years & 125,984 & 8188 & 2549 & & 37,292 & 3401 & 1413 & \\
\hline No. of cases & 156 & 21 & 14 & & 184 & 14 & 11 & \\
\hline \multirow[t]{2}{*}{ Multivariate RR (95\% CI) } & 1.0 & $1.7(1.0-2.6)$ & $3.1(1.8-5.5)$ & $<0.001$ & 1.0 & $0.7(0.4-1.2)$ & $1.2(0.7-2.3)$ & 0.94 \\
\hline & \multicolumn{4}{|c|}{ Lower BMI } & \multicolumn{4}{|c|}{ Higher BMI } \\
\hline Person-years & 82,087 & 5569 & 1620 & & 81,189 & 6021 & 2341 & \\
\hline No. of cases & 153 & 13 & 8 & & 187 & 22 & 17 & \\
\hline \multirow[t]{2}{*}{ Multivariate RR (95\% CI) } & 1.0 & $0.9(0.5-1.6)$ & $1.9(0.9-3.9)$ & 0.21 & 1.0 & $1.2(0.8-1.9)$ & $2.2(1.3-3.6)$ & 0.004 \\
\hline & \multicolumn{4}{|c|}{ Lower TSF } & \multicolumn{4}{|c|}{ Higher TSF } \\
\hline Person-years & 69,608 & 4163 & 1553 & & 93,668 & 7426 & 2408 & \\
\hline No. of cases & 155 & 10 & 8 & & 185 & 25 & 17 & \\
\hline \multirow[t]{2}{*}{ Multivariate RR (95\% CI) } & 1.0 & $0.9(0.5-1.7)$ & $1.4(0.7-2.9)$ & 0.53 & 1.0 & $1.2(0.8-1.8)$ & $2.3(1.4-3.9)$ & 0.002 \\
\hline & \multicolumn{4}{|c|}{ Lower SSF } & \multicolumn{4}{|c|}{ Higher SSF } \\
\hline Person-years & 79,092 & 4927 & 1592 & & 84,185 & 6662 & 2370 & \\
\hline No. of cases & 174 & 11 & 5 & & 166 & 24 & 20 & \\
\hline \multirow[t]{2}{*}{ Multivariate RR (95\% CI) } & 1.0 & $0.7(0.4-1.4)$ & $0.8(0.3-2.0)$ & 0.44 & 1.0 & $1.4(0.9-2.1)$ & $2.8(1.8-4.5)$ & $<0.001$ \\
\hline & \multicolumn{4}{|c|}{ Non-hypertensive with lower BMI } & \multicolumn{4}{|c|}{ Non-hypertensive with higher BMI } \\
\hline Person-years & 68,644 & 4380 & 1140 & & 57,340 & 3809 & 1409 & \\
\hline No. of cases & 78 & 9 & 5 & & 78 & 12 & 9 & \\
\hline \multirow[t]{2}{*}{ Multivariate RR (95\% CI) } & 1.0 & $1.5(0.7-3.0)$ & $2.5(1.0-6.3)$ & 0.03 & 1.0 & $1.8(0.9-3.3)$ & $3.6(1.8-7.4)$ & $<0.001$ \\
\hline & \multicolumn{4}{|c|}{ Hypertensive with lower BMI } & \multicolumn{4}{|c|}{ Hypertensive with higher BMI } \\
\hline Person-years & 13,443 & 1189 & 481 & & 23,849 & 2212 & 933 & \\
\hline No. of cases & 75 & 4 & 3 & & 109 & 10 & 8 & \\
\hline \multirow[t]{2}{*}{ Multivariate RR (95\% CI) } & 1.0 & $0.5(0.2-1.5)$ & $1.0(0.3-3.2)$ & 0.51 & 1.0 & $0.8(0.4-1.6)$ & $1.5(0.7-3.1)$ & 0.60 \\
\hline & \multicolumn{4}{|c|}{ Non-hypertensive with lower TSF } & \multicolumn{4}{|c|}{ Non-hypertensive with higher TSF } \\
\hline Person-years & 55,978 & 3116 & 1047 & & 70,006 & 5073 & 1501 & \\
\hline No. of cases & 72 & 8 & 5 & & 84 & 13 & 9 & \\
\hline \multirow[t]{2}{*}{ Multivariate RR (95\% CI) } & 1.0 & $1.5(0.7-3.1)$ & $3.0(1.1-7.8)$ & 0.01 & 1.0 & $1.8(1.0-3.3)$ & $3.6(1.7-7.4)$ & $<0.001$ \\
\hline & \multicolumn{4}{|c|}{ Hypertensive with lower TSF } & \multicolumn{4}{|c|}{ Hypertensive with higher TSF } \\
\hline Person-years & 13,631 & 1048 & 507 & & 23,662 & 2354 & 907 & \\
\hline No. of cases & 83 & 2 & 3 & & 101 & 12 & 8 & \\
\hline \multirow[t]{2}{*}{ Multivariate RR (95\% CI) } & 1.0 & $0.4(0.1-1.5)$ & $0.6(0.2-2.1)$ & 0.90 & 1.0 & $0.9(0.5-1.7)$ & $1.8(0.9-3.8)$ & 0.26 \\
\hline & Non-hyp & tensive with lo & ver SSF & & Non-hyp & rtensive with $\mathrm{h}$ & gher SSF & \\
\hline Person-years & 64,990 & 3760 & 1083 & & 60,994 & 4428 & 1465 & \\
\hline No. of cases & 88 & 9 & 3 & & 68 & 12 & & \\
\hline Multivariate RR $(95 \% \mathrm{CI})$ & 1.0 & $1.3(0.7-2.7)$ & $1.5(0.5-5.0)$ & 0.23 & 1.0 & $1.9(1.0-3.7)$ & $4.9(2.5-9.5)$ & $<0.001$ \\
\hline & Hyperten & ive with lower & SF & & Hyperten & ive with higher & SSF & \\
\hline Person-years & 14,102 & 1167 & 509 & & 23,191 & 2234 & 905 & \\
\hline No. of cases & 86 & 2 & 2 & & 98 & 12 & 9 & \\
\hline Multivariate RR (95\% CI) & 1.0 & $0.3(0.1-1.1)$ & $0.4(0.1-1.6)$ & 0.05 & 1.0 & $1.0(0.5-1.9)$ & $1.8(0.9-3.7)$ & 0.17 \\
\hline
\end{tabular}

Multivariate adjustments: sex and the same variables listed in Table 2, except for the stratified variable. Adiposity category was divided by the sex-specific median value: BMI,

those with higher values for SSF, which is an indicator of central obesity. Compared with those in the corresponding subgroups of the normal glucose category, diabetic non-hypertensive subjects with higher BMI or TSF values had a four-fold increased risk of non-embolic ischaemic stroke, and diabetic non-hypertensive
$22.7 \mathrm{~kg} / \mathrm{m}^{2}$ for men and $23.1 \mathrm{~kg} / \mathrm{m}^{2}$ for women; TSF, $6 \mathrm{~mm}$ for men and $15 \mathrm{~mm}$ for women; and SSF, $11 \mathrm{~mm}$ for men and $18 \mathrm{~mm}$ for women

subjects with higher SSF values had a five-fold increased risk. Non-hypertensive subjects with higher values for measures of adiposity and borderline diabetes had a two-fold increased risk compared with those in the corresponding subgroups of the normal glucose category. The strong association between glucose ab- 

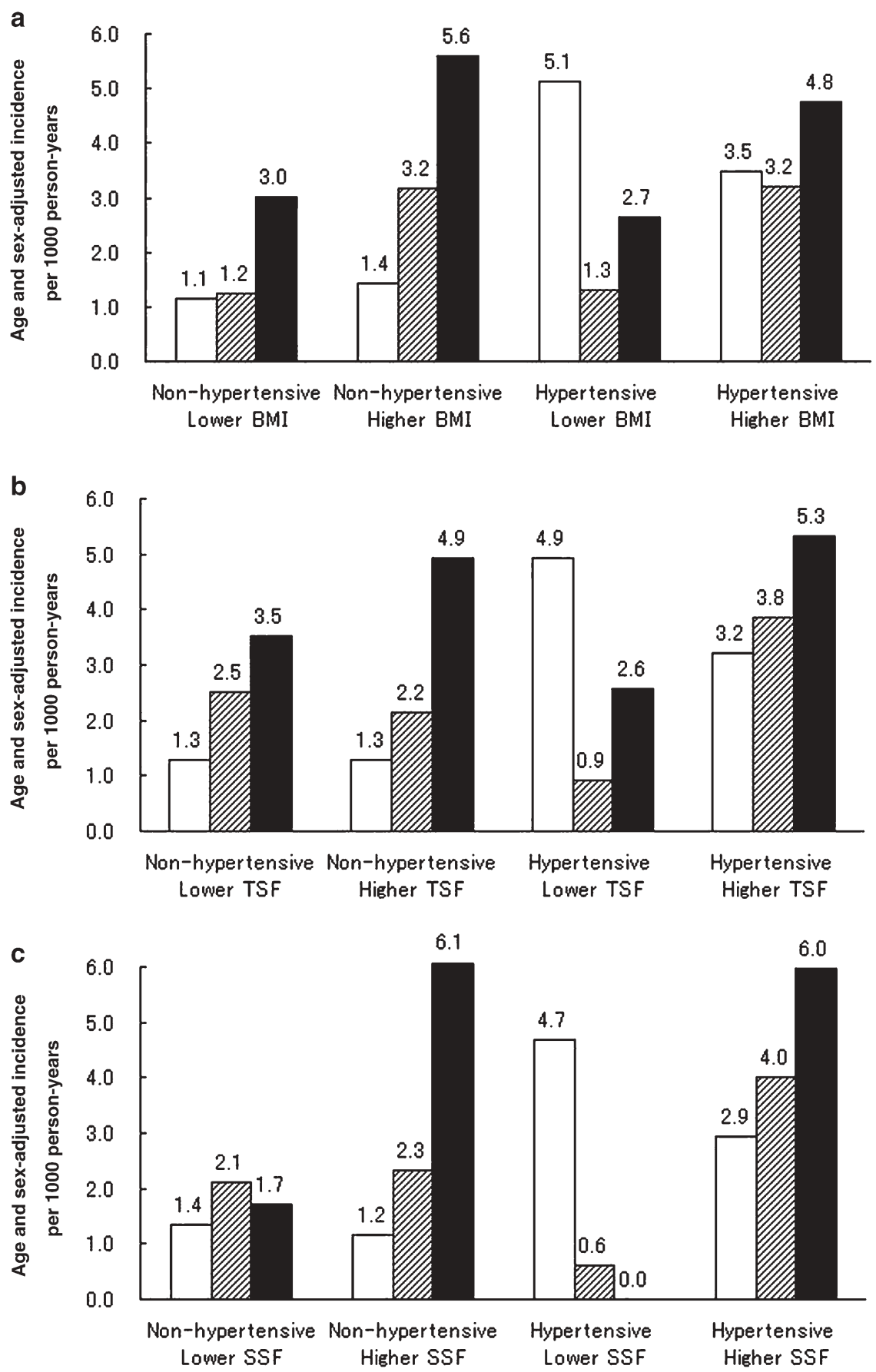

Fig. 1. Age- and sex-adjusted incidence of non-embolic ischaemic stroke (per 1000 person-years) according to serum glucose category stratified by hypertension and (a) BMI, (b) TSF and (c) SSF. Open bars, normal glucose category; hatched bars, borderline diabetes catogory; filled bars, diabetes category

normality and risk of ischaemic stroke in non-hypertensive subjects with higher adiposity was explained in part by the higher glucose levels observed in the diabetic patients in this subgroup compared with those in diabetic subjects in other subgroups. This finding suggests that the diabetic subjects in the non-hypertensive/higher adiposity subgroup had more advanced diabetes. Secondly, the susceptibility of non-hypertensive subjects to glucose abnormalities may be indicative of non-hypertensive mechanisms (e.g. microvascular disease) linked to ischaemic stroke. The fact that a greater proportion of the ischaemic stroke cases were lacunar strokes (64\%) rather than large-artery occlusive infarctions suggests that this may be the case. In hypertensive subjects, glucose abnormalities may not play a prominent role in the pathogenesis of stroke because hypertension is the predominant risk factor for stroke $[3,7]$. Alternatively, it is possible that this finding was due to chance alone. To rule out this possibility, further studies are required to confirm the findings of these subgroup analyses. 
In our study population, the proportion of strokes attributable to diabetes was only $2.5 \%$ in men and $2.3 \%$ in women, due to the low prevalence of diabetes (3.2\% in men and $2.0 \%$ in women). These estimates increased to $10 \%$ for men and $8 \%$ for women when we used the recently published prevalence rates of diabetes in Japan $(13.4 \%$ in men and $6.8 \%$ in women aged 40-69 years [14]). However, these figures are still low compared with those reported for Western countries (20-30\%) where the prevalence of diabetes in middle-aged and older adults is approximately $20 \%$ $[1,2,3]$. This implies that the public health burden of diabetes-associated stroke is still lesser in Japan than it is in Western countries.

However, in Japan, the mean BMI consistently increased in men between 1970 and 2000, according to a national survey [15], and population-based surveys have revealed that the prevalence of diabetes has increased in both men and women [5]. Conversely, blood pressure levels decreased in both men and women between 1970 and 2000, which contributed to a decline in stroke mortality [15]. These trends may increase the relative importance of diabetes in the development of stroke in the Japanese population.

In the present study, lacunar infarctions comprised $64 \%$ of non-embolic ischaemic strokes and diabetes doubled the risk of lacunar infarction in both men and women. Our finding adds to existing evidence that diabetes raises the risk of lacunar infarction in both sexes, since a previous Japanese study showed a significant independent association only among men [4]. It is plausible that glucose abnormality or insulin resistance causes microangiopathy, with arteriosclerosis in small penetrating arterioles of the cerebral basal ganglia, leading to lacunar infarction [16]. We were not able to examine the relationship between glucose abnormality and risk of large-artery occlusive infarction because of the small number of cases ( $n=1$ among diabetic participants). The high proportion of largeartery occlusive infarctions among ischaemic strokes (approximately 60-70\%) [17], and the positive association between diabetes and the risk of ischaemic stroke among Western populations, suggest that diabetes also increases the risk of large-artery occlusive infarction. The results of a retrospective, case-controlled study indicate that this is also the case in the Japanese population [18].

The strengths of the present study include the large population-based sample of middle-aged Japanese men and women, and the use of standardised methods for the measurement of serum glucose and cardiovascular risk factors. Data collection on incident strokes was complete, and a high percentage of events were confirmed using imaging studies. These study characteristics allowed us to conduct analyses for ischaemic stroke subtype (lacunar infarction) and permitted stratification of subjects according to variable constitutional factors (i.e. hypertension and adiposity).
One of the limitations associated with the present study is that we relied on a single glucose measurement, and did not require the presence of symptoms in persons with a non-fasting glucose level $\geq 11.1 \mathrm{~mol} / \mathrm{l}$. Our classification system is more likely to identify false positives in terms of diabetes, thus weakening the association between diabetes and risk of ischaemic stroke. Consequently, the true association would be stronger than that reported in the present study.

Furthermore, we did not measure fasting serum insulin at baseline, which meant that the relationship between insulin resistance and risk of ischaemic stroke in non-diabetic individuals could not be examined. Two previous cohort studies of whites showed a significant independent relationship between serum insulin levels and risks of total [19] and ischaemic [20] strokes among non-diabetic individuals, while other studies in whites $[20,21]$ did not show an independent relationship.

In conclusion, in the present study, diabetes was a significant risk factor for non-embolic ischaemic stroke in Japanese men and women, particularly non-hypertensive and non-lean individuals. In Japan, there has been a nationwide decrease in blood pressure levels in both men and women, and an increase in BMI in men over the last three decades. If the nationwide prevalence of diabetes increases in Japan, as indicated by population-based surveys [5], the impact of glucose abnormality on the absolute risk of ischaemic stroke may increase and thereby become a public health issue.

Acknowledgements. The authors thank A.R. Folsom (Division of Epidemiology, University of Minnesota, Minneapolis, Minn., USA) for his valuable comments. This study was supported in part by research grants (numbers 10150306 and 13071901) from the Japanese Ministry of Health, Welfare and Labour. The authors are not aware of any conflict of interest in this study.

\section{References}

1. Abbott R, Donahue RP, MacMahon SW et al. (1987) Diabetes and the risk of stroke. The Honolulu Heart Program. JAMA 257:949-952

2. Folsom AR, Rasmussen ML, Chambless LE et al. (1999) Prospective associations of fasting insulin, body fat distribution, and diabetes with risk of ischemic stroke. The Atherosclerosis Risk in Community (ARIC) Study Investigators. Diabetes Care 22:1077-1083

3. McCarron P, Greenwood R, Elwood P et al. (2001) The incidence and aetiology of stroke in the Caerphilly and Speedwell Collaborative Studies II: risk factors for ischemic stroke. Public Health 115:12-20

4. Tanizaki Y, Kiyohara Y, Kato I et al. (2000) Incidence and risk factors for subtypes of cerebral infarction in a general population. The Hisayama Study. Stroke 31:2616-2622

5. Ohmura T, Ueda K, Kiyahara Y et al. (1993) Prevalence of Type 2 (non-insulin-dependent) diabetes mellitus and impaired glucose tolerance in the Japanese general population: the Hisayama Study. Diabetologia 36:1198-1203 
6. Ueshima H, Tatara K, Asakura S, Okamoto M (1987) Declining trends in blood pressure level and the prevalence of hypertension, and changes in related factors in Japan, 1956-1980. J Chronic Dis 40:137-147

7. Shimamoto T, Komachi Y, Inada H et al. (1989) Trends for coronary heart disease and stroke and their risk factors in Japan. Circulation 79:503-515

8. Iso H, Rexrode K, Hennekens CH et al. (2000) Application of computer tomography-oriented criteria for stroke subtype classification in a prospective study. Ann Epidemiol 10:81-87

9. Kitamura A, Shimamoto T, Doi M et al. (1991) Secular trends in prevalence and incidence of atrial fibrillation and associated factors in a Japanese rural population. Nippon Koshu Eisei Zasshi 38:95-105

10. Kuzuya T, Nakagawa S, Satoh J et al. (2002) Report of the Committee on the Diagnostic Criteria of Diabetes Mellitus. Diabetes Res Clin Pract 55:65-85

11. The Expert Committee on the Diagnosis and Classification of Diabetes Mellitus (1997) Report of the Expert Committee on the Diagnosis and Classification of Diabetes Mellitus. Diabetes Care 20:1183-1197

12. Nakamura M, Morita M, Yabuuchi E et al. (1982) The evaluation and the results of cooperative cholesterol and triglyceride standardization program by WHO-CDC [article in Japanese]. Risho Byori 30:325-332

13. Kirkendall WM, Feinlieb M, Freis ED et al. (1980) Recommendations for human blood pressure determination by sphygmomanometers. Subcommittee of the AHA Postgraduate Education Committee. Circulation 62:1146A-1155A
14. The Ministry of Health, Welfare and Labour (2003) The National Survey of Diabetes 2002 [article in Japanese]. Available from http://www.mhlw.go.jp/shingi/2003/08/ s0806-4.html, accessed 1 November 2004

15. Nutrition Division Public Health Bureau, The Ministry of Health and Welfare (1999) Kokumin Eiyo no Genjyo (The National Nutrition Survey) [article in Japanese]. Daiichi Shuppan, Tokyo

16. Zunker P, Schick A. Buschmann HC et al. (1996) Hyperinsulinism and cerebral microangiopathy. Stroke 27:219-223

17. Tanaka H, Iso H, Yokoyama T et al. (2001) Cerebrovascular disease. In: Detels R, McEwen J, Beaglehole R, Tanaka $\mathrm{H}$ (eds) Oxford textbook of public health, vol 3, 4th rev edn. Oxford University Press, London, pp 1193-1226

18. Shinozaki K, Naritomi H, Shimizu T et al. (1996) Role of insulin resistance associated with compensatory hyperinsulinemia in ischemic stroke. Stroke 27:37-43

19. Lakka H-M, Lakka TA, Tuomilehto J, Sivenius J, Salonen JT (2000) Hyperinsulinemia and the risk of cardiovascular death and acute coronary and cerebrovascular events in men. The Kuopio Ischaemic Heart Disease Risk Factor Study. Arch Intern Med 160:1160-1168

20. Pyorala M, Miettinen H, Laakso M et al. (1998) Hyperinsulinemia and the risk of stroke in healthy middle-aged men. The 22-year follow-up results of the Helsinki Policemen Study. Stroke 29:1860-1866

21. Wannamethee SG, Perry IJ, Shaper AG (1999) Nonfasting serum glucose and insulin concentrations and the risk of stroke. Stroke 30:1780-1786 